Roger Keller Celeste ${ }^{1}$

Jacqueline Furtado Vital ${ }^{1}$

Washington Leite Junger ${ }^{1}$

Michael Eduardo Reichenheim ${ }^{1}$

${ }^{1}$ Departamento de

Epidemiologia, Instituto de Medicina Social,

Universidade Estadual do Rio de Janeiro. Rua São Francisco Xavier 524/70 Bls. D e E. 20550-900 Rio de Janeiro RJ.

rk_celeste@hotmail.com

\section{Séries de procedimentos odontológicos realizadas nos serviços públicos brasileiros, 1994-2007}

\author{
Time series analysis of dental care procedures \\ in Brazilian public services, 1994-2007.
}




\section{Introdução}

A influência dos serviços de saúde odontológicos na saúde bucal da população tem sido considerada limitada ${ }^{1}$. Entretanto, essa influência pode ser positiva dependendo do conjunto de procedimentos realizados e do resultado esperado ${ }^{2,3}$. Assim, o estudo de tendências de procedimentos específicos (e.g. taxa de consultas odontológicas, de procedimentos preventivos ou exodontias) pode ajudar a esclarecer os efeitos que os serviços de saúde podem ter na saúde bucal da população, bem como a importância relativa de cada procedimento. Tendências no uso dos serviços odontológicos têm sido relatadas em países desenvolvidos e apontam para um aumento nos Estados Unidos, Reino Unido, Países Escandinavos, Austrália e Nova Zelândia ${ }^{4}$. Nestes países, houve um aumento nas visitas para checkup e prevenção e uma diminuição nos procedimentos como extrações e próteses ${ }^{4-7}$.

No Brasil, escassa evidência sobre tendências foi encontrada. Pinheiro e Torres ${ }^{8}$ mostraram que o percentual de brasileiros que nunca consultaram um dentista diminuiu de $18,7 \%$ para $15,9 \%$ entre 1998 e 2003, utilizando dados da Pesquisa Nacional por Amostra Domiciliar (PNAD). Dois estudos avaliaram as séries temporais anuais de diversos procedimentos realizados no Sistema Único de Saúde (SUS) em três municípios brasileiros e encontraram uma grande flutuação nas taxas ao longo dos anos estudados, não permitindo conclusões sobre a existência de tendências ${ }^{9,10}$.

Pouco se sabe, também, sobre o padrão e os determinantes dessas séries. O comportamento delas, no entanto, deve revelar os determinantes de utilização dos serviços, os quais podem ser resumidos em: a) padrão da doença; b) características dos prestadores de serviços; c) características individuais dos usuários; d) forma de organização dos serviços e; e) política do sistema de saú$\mathrm{de}^{11}$. Técnicas que permitem o estudo separado dos componentes das séries temporais (e.g. tendência, sazonalidade, ciclos e ruído branco) podem fornecer valiosas informações sobre os determinantes de uso e acesso dos serviços, em especial para avaliar o impacto de políticas públicas.

A política de descentralização da saúde, conhecida como municipalização, fez com que os municípios assumissem a gestão local e a execução dos serviços e ações de saúde ${ }^{12}$. Uma forma de avaliação do processo de municipalização é através do percentual de municípios habilitados, por exemplo, em 1999, 97\% dos municípios estavam habilitados em algum tipo de gestão ${ }^{13}$. Entretanto, a avaliação da municipalização deve ter como um dos seus atributos fundamentais o monitoramento da cobertura populacional das ações em saúde ${ }^{14}$. Desde o início desse processo, pode-se esperar um aumento da cobertura populacional por dois motivos. Primeiramente, porque houve um aumento no percentual de municípios habilitados. Em segundo, houve estratégias para a redução de subnotificação, como a Portaria 1882, a qual estabeleceu que no caso de não alimentação dos sistemas de informações a transferência de recursos financeiros para os municípios ficaria suspensa ${ }^{15}$.

Em saúde bucal, a atual política ministerial tem estimulado a inclusão das Equipes de Saúde Bucal (ESB) nas de saúde da família, como forma de expandir o acesso e reestruturar a atenção básica, incluindo atividades extraclínica (e.g.,visitas domiciliares) e dando menos ênfase a atividades mutiladoras, como as exodontias ${ }^{16,17}$. Em dezembro de 2000, foram inseridas ações de saúde bucal no Programa de Saúde da Família (PSF), sendo a Equipe de Saúde Bucal (ESB) na modalidade 1 composta por um(a) Cirurgião-Dentista (CD) e um(a) Auxiliar de Saúde Bucal (ASB), e na modalidade 2 a essa equipe é acrescentada um(a) Técnico em Saúde Bucal (TSB). Segundo a Coordenação Nacional de Saúde Bucal ${ }^{18}$, na segunda metade de 2007, as ESB-PSF estavam atuantes em 4285 municípios brasileiros com uma estimativa de cobertura de $68 \%$ da população. Em 2005, estimou-se que $27,3 \%$ dos CD que trabalhavam na rede pública estavam nas ESB-PSF ${ }^{19}$ e entre $85,8 \%$ e $91 \%$ dos profissionais das ESB-PSF trabalhavam em regime de 40 horas semanais ${ }^{19,20}$. Portanto, considerando-se tais informações, é de se esperar um aumento expressivo na taxa total de procedimentos notificados após o ano de 2000. Relatos sobre os impactos das ESB no acesso aos serviços de saúde têm sido descritos na região norte $^{21}$ e nordeste ${ }^{22,23}$, mas os resultados não são divergentes e não há evidência em nível nacional até o momento.

Assim, o presente trabalho se propõe a descrever o padrão temporal de taxas mensais de procedimentos odontológicos no SUS, entre 1994 e 2007, avaliando o possível impacto de políticas de saúde bucal. Um segundo objetivo é descrever e avaliar se há diferença no padrão das taxas de acesso aos serviços entre as macrorregiões brasileiras. 


\section{Métodos}

Trata-se de um estudo ecológico, adotando-se o Brasil e suas cinco macro-regiões como unidades de observação. As fontes para obtenção dos dados analisados foram: o Sistema de Informação Ambulatorial (SIA-SUS) do Ministério da Saúde no período de julho de 1994 a julho 2007; os censos de 1991 e de 2000; e a contagem da população de 1996. Todos os dados foram provenientes do Sistema de Informações Ambulatoriais do DATASUS.

Foram calculadas cinco taxas mensais de procedimentos odontológicos por mil habitantes tendo o Brasil como unidade de agregação: a) procedimentos odontológicos coletivos (PC); b) procedimentos odontológicos preventivos (PP); c) exodontias (PE); d) procedimentos restauradores (PR) e; e) taxa total de procedimentos odontológicos (PO). A taxa total de procedimentos, PO, também foi calculada para cada macrorregião brasileira. O numerador de cada taxa foi obtido somando o número de procedimentos realizados em cada mês, enquanto que o denominador foi a população residente no mês correspondente. A população residente em cada mês foi estimada com base no Método da Progressão Geométrica a partir dos dados censitários ${ }^{24}$.

\section{Procedimentos analisados}

A tabela de códigos de procedimentos do SIASUS teve alteração de nomenclatura e códigos em outubro de $1999^{25}$. Assim, para o período de julho de 1994 até outubro de 1999 foram somados, para cada taxa, os códigos:

Total de procedimentos: 05 -atendimento odontológico (procedimentos PAB), 25-atendimento odonto. (procedimentos não $\mathrm{PAB}$ )

Restaurações: 242-restauração a pino (PAB); 234-restauração c/amalgama duas/mais faces (PAB); 236-restauração com amalgama de uma face (PAB); 230-restauração compósito duas ou mais faces (PAB); 220-restauração compósito de uma face (PAB); 222-restauração compósito envolvendo ângulo incisal (PAB); 238-restauração c/ silicato duas ou mais faces (PAB); 239-restauração com silicato de uma face (PAB); 244-restauração fotopolimerizável duas ou mais faces (PAB); 246-restauração fotopolimerizável uma face (PAB); 221-Dentística I; 223-Dentística II

Exodontias: 247-exodontia de dente permanente (PAB), 224-exodontia de dente decíduo (PAB), 241-odontologia cirúrgica I, 225-remoção de resto radicular (PAB).
Procedimentos preventivos: 200-aplicação terap. intensiva c/flúor p/sessão (PAB), 206-escariação por dente (PAB), 209-odontologia preventiva I, 210-controle da placa bacteriana (PAB), 211-odontologia preventiva II, 212-aplicação de selante por dente (PAB), 215-odontologia preventiva IV, 217-odontologia preventiva V, 232aplicação cariostático por dente (PAB)

Procedimentos coletivos: 201-procedimentos coletivos II (PC II) 203-procedimentos coletivos III (PC III)

Para o período de novembro de 1999 até julho de 2007 foram somados os códigos:

Total de procedimentos: Grupos: 03-Ações Básicas Em Odontologia e 10-Ações Especializadas Em Odontologia.

Restaurações: 0303103-restauração a pino; 0303104-restauração com amalgama duas/mais faces; 0303105-restauração com amalgama uma face; 0303106-restauração com compósito duas ou mais faces; 0303107-restauração com compósito uma face; 0303108-restauração com compósito envolvendo ângulo incisal; 0303109-restauração com silicato duas/mais faces; 0303110restauração com silicato uma face; 0303111-restauração fotopolimerizável 2/mais faces; 0303112-restauração fotopolimerizável uma face; 1001101-restauração com ionômero vidro duas/ mais faces 1001102-restauração com ionômero vidro uma face.

Exodontias: 0304101-exodontia de dente decíduo, 0304102-exodontia de dente permanente, 0304103-remoção de resto radicular e 1005114exodontia múltipla com alveoloplastia por hemiarco.

Procedimentos preventivos: 0302201-aplicação terapêut. intensiva c/ flúor-p/sessão, 0302202aplicação de cariostático por dente, 0302203-aplicação de selante por dente, 0302204-controle da placa bacteriana, 0302205-escariação por dente.

Procedimentos coletivos: 0301101 -procedimentos coletivos (paciente/mês), 0301102-ação coletiva escovação dental supervisionada, 0301103-ação coletiva de bochecho fluorado, 0301104-ação coletiva aplicação tópica de flúor gel, 0301105-ação colet. exame bucal.

\section{Análise de tendência}

As tendências brutas e suavizadas das taxas de PP, PC, PR, PE e PO do Brasil e as taxas de PO das cinco macrorregiões foram descritas graficamente. A suavização, para remoção de sazonalidade, foi feita por meio de médias móveis de 12 meses ${ }^{26}$. Tal procedimento, não paramétrico, per- 
mite averiguar visualmente eventos acíclicos, que alteram a tendência de uma série.

\section{Avaliação de políticas federais (intervenções) nas tendências de notificação}

Foram analisadas alterações de tendência de notificação do registro de uso/acesso dos serviços em três momentos da série: janeiro de 1998, outubro de 1999 e dezembro de 2000.

O primeiro momento para a possível alteração da série, janeiro de 1998, foi decorrência da Portaria 1882/GM de 18 de dezembro de $1997^{15} \mathrm{e}$ da Instrução Normativa 01/98 de janeiro de $1998^{27}$. Ambas promoveram um avanço no processo de descentralização da gestão do sistema de saúde, forçando os municípios a assumirem a assistência à saúde e responsabilizando os mesmos pela alimentação do SIA-SUS. Nesse momento também houve a alteração da nomenclatura dos códigos do SIA-SUS, muitos procedimentos sendo desdobrados ou excluídos ${ }^{28}$. Os procedimentos anteriores a essa data foram considerados “procedimentos não PAB". Como não há uma tabela para consulta com a equivalência de códigos, a conversão foi feita de forma heurística com base na migração dos procedimentos. Por exemplo, o total de exodontias a partir de janeiro de 1998 corresponde, grosso modo, ao total de procedimentos antes considerados "Odontologia Cirúrgica I" e as restaurações correspondem a "Dentística I" e "Dentística II". Já os procedimentos coletivos (PC-II e PC-III) ficaram sem correspondente.

O segundo momento de análise foi em outubro de 1999, quando houve uma alteração da nomenclatura dos códigos do SIA-SUS e inclusão de novos procedimentos ${ }^{25}$. A existência de uma tabela de conversão permite a comparabilidade com procedimentos anteriores, mas a criação de novos procedimentos poderia aumentar o número total de notificações.

Por fim, dezembro de 2000 foi a terceira medida que poderia alterar o número de procedimentos odontológicos notificados. A Portaria $1.444^{29}$ estabeleceu incentivo financeiro para inclusão da equipe de saúde bucal no PSF.

Procedeu-se, então, com a análise de intervenção usando a metodologia proposta por Box e Tiao $^{30}$. Primeiro, foram criadas três variáveis indicadoras para os períodos de intervenção referentes a janeiro de 1998, outubro 1999 e dezembro de 2000. Estas variáveis são compostas de valores 0 (zero) para o período anterior à in- tervenção e 1 (um) para o período a partir da intervenção. Para cada série temporal (exceto para taxas de PO das macro-regiões) foi ajustado um modelo SARIMA ${ }^{31}$ para o período anterior à primeira intervenção a fim de se definir o processo de fundo (background), e as variáveis indicadoras das intervenções foram introduzidas no modelo via função de transferência ${ }^{31}$. Desta forma é possível avaliar o impacto de cada intervenção no nível da série temporal. Importante ressaltar que as tendências existentes a cada intervenção foram removidas, de forma a avaliar se a intervenção específica produziu uma mudança no nível das séries.

Foram usados modelos capazes de dar conta da sazonalidade das séries temporais (SARIMA ${ }^{31}$ ). Os seguintes diagnósticos dos resíduos foram usados para avaliar a adequação dos modelos antes e após as intervenções: periodograma, gráfico de normalidade, função de autocorrelação, AIC (Akaike Information Criterion) e teste de Ljung-Box ${ }^{31}$. As séries foram diferenciadas implicitamente, ARIMA ( $\mathrm{p}, 1, \mathrm{q})$, quando necessário para garantir a estacionaridade da série temporal e, portanto, os interceptos nos modelos para as séries diferenciadas foram igual a zero. Para séries não estacionárias, o intercepto para o mês imediatamente anterior à intervenção foi estimado por regressão linear das séries suavizadas até o momento da primeira intervenção.

As séries foram modeladas na sua escala natural, ou seja, taxas. Para interpretar o efeito das intervenções, é necessário considerar os efeitos das intervenções anteriores. Assim, o coeficiente da variável indicadora referente ao PSF representa o acréscimo marginal no nível do processo considerando as intervenções anteriores, uma vez que as intervenções foram mudanças de caráter definitivo, ou seja, não houve regresso à situação anterior.

\section{Análise de períodos cíclicos e sazonalidade}

Apenas as séries de taxas de PO de cada macro-região brasileira foram descritas em termos de padrões cíclicos.

Diferentes métodos foram usados para análise do padrão repetitivo das séries temporais. No domínio do tempo, foi descrita a estrutura das séries de PO pela Função de Autocorrelação (ACF), representada graficamente pelo correlograma. Essa avaliação permite que se identifique a correlação entre as observações atuais de uma série com períodos (lags) em diferentes defasa- 
gens de tempo, como por exemplo, padrões sazonais expressando correlação mais alta entre observações espaçadas de 12 meses no tempo. Outra forma utilizada foi a avaliação de frequência de ondas senoidais, expressas através do periodograma. Nessa avaliação, a série é decompostas em várias ondas senoidais com diferentes frequências (i.e. períodos mensais), sendo que algumas dessas irão melhor explicar a variabilidade dos dados.

\section{Análise do componente residual}

Para todas as séries, procedeu-se com a remoção da tendência das mesmas pelo método de diferenciação de primeira ou segunda ordem e remoção da sazonalidade por meio de médias móveis ${ }^{26}$. Então, cada série foi testada para avaliar se o componente restante era compatível com uma série de ruído branco utilizando-se o teste de Portmanteau ${ }^{26}$.

Todas as análises foram realizadas nos softwares estatísticos Stata 9.2 e R.

\section{Resultados}

Cada série temporal completa constituiu-se de 157 meses. As taxas de exodontias, restaurações, procedimentos coletivos e preventivos representaram juntas em média 73,9\% (mês mínimo = $60,9 \% \%$; mês máximo $=79,9 \%$ ) do total de procedimentos. Os procedimentos coletivos foram responsáveis por $31,1 \%$ do total ( mínimo $=0 \%$; máximo $=47,8 \%)$, os procedimentos preventivos por $16,4 \%($ mínimo $=13,1 \%$; máximo $=$ $28,4 \%$ ), as restaurações por $17,8 \%$ (mínimo $=$ $10,8 \%$; máximo $=29,9 \%$ ) e as exodontias por $8,5 \%($ mínimo $=5,1 \% ;$ máximo $=14,1 \%)$.

\section{Tendências}

As séries brutas e suavizadas são apresentadas nas Figuras 1 e 2, nas quais os três momentos estudados estão indicador por linhas verticais. Percebe-se que houve uma elevação de nível nas taxas de PO entre janeiro de 1998 e janeiro de 2000 no Brasil como um todo (Figura 1). O mesmo padrão é observado nas regiões Sul, Sudeste e Centro-Oeste (Figura 2), mas após dezembro de 2000 essas regiões apresentaram uma tendência de decrescimento que não foi estatisticamente significante. Na região Nordeste houve um significativo aumento gradual na série de $\mathrm{PO}$ entre janeiro de 1998 e 2007, que não foi alterada em
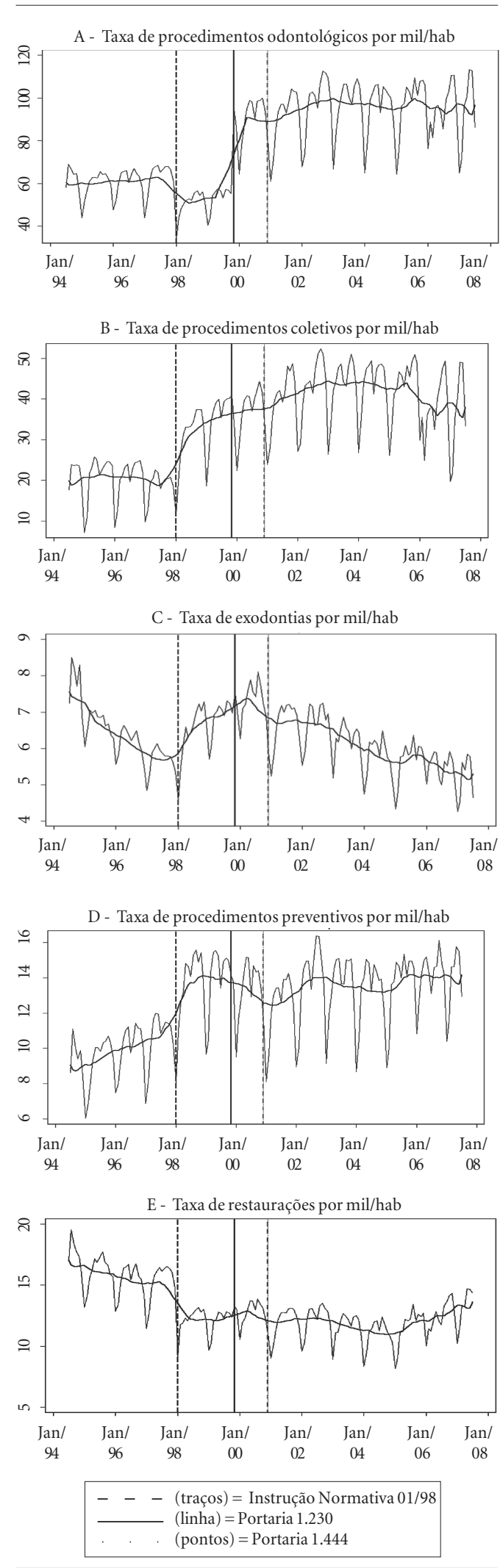

Figura 1. Taxas mensais de diferentes procedimentos odontológicos por mil/habitantes com linha de tendência suavizada, no Brasil, entre julho 1994 a julho 2007. 

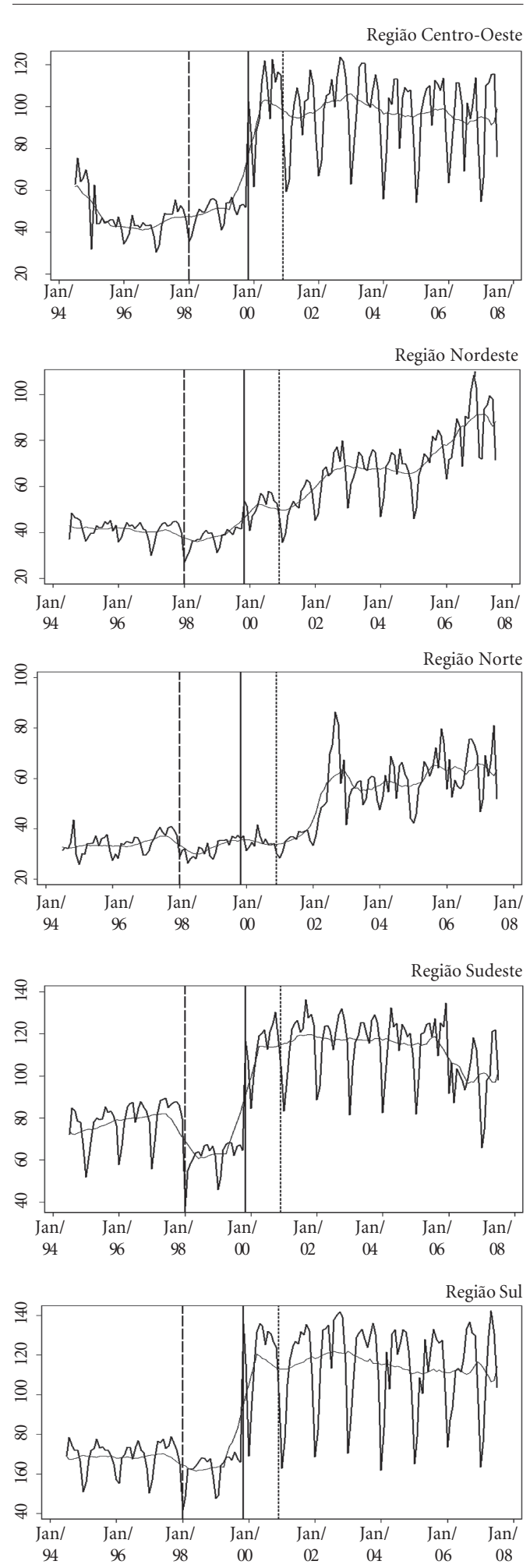

- _ $\quad$ - $($ traços $)=$ Instrução Normativa $01 / 98$

- (linha) $=$ Portaria 1.230

(pontos) $=$ Portaria 1.444

Figura 2. Taxas mensais totais de procedimentos odontológicos por mil/habitantes com linhas de tendência suavizadas, nas cinco macro-regiões brasileiras, entre julho 1994 a julho 2007. dezembro de 2000. Na região Norte foi observado um significativo aumento nas taxas de PO apenas após dezembro de 2000. Tendências nacionais de procedimentos específicos (Figura 1), PC, PP, PR e PE apresentaram comportamentos distintos entre si. Visualmente, PC, PR e PP mostraram-se constantes após janeiro de 2000, enquanto PE declinou através de todo período, com uma elevação de nível entre janeiro de 1998 e janeiro de 2000.

As taxas suavizadas de PO, PR e PE mostraram-se estacionárias após diferenciação de primeira ordem e compatíveis com uma série de ruído branco. Por outro lado, as taxas suavizadas de PC e PP apresentaram-se estacionárias após diferenciação de segunda ordem.

Os coeficientes de regressão SARIMA (Tabela 1) expressam o efeito de cada intervenção (jan/ 98, out/99 e dez/2000) na tendência das séries. A partir de janeiro de 1998 houve uma mudança estatisticamente significante da tendência existente em todas as taxas de procedimentos exceto na taxa de exodontias. Os resultados após a alteração da nomenclatura (outubro de 1999) e após a portaria (dezembro de 2000) que incentivou a inclusão da Equipe de Saúde Bucal no PSF (ESBPSF) variaram de acordo com o tipo de procedimento observado.

\section{Sazonalidade}

As séries de $\mathrm{PO}$ apresentaram um padrão cíclico marcante nas regiões Centro-Oeste, Sudeste e Sul, o que configura o ciclo sazonal (Figura 2). Comportamento semelhante é encontrado na região Nordeste, porém com um nível menor. Finalmente, a região Norte apresenta um padrão similar à região Nordeste até outubro de 1999, com alteração no comportamento da série em 2003.

A análise de sazonalidade nas taxas de PO pelos correlogramas e periodograma mostrou que todas as macrorregiões apresentam flutuações sazonais de 6 em 6 meses e de 12 em 12 meses, com exceção da região Norte. A intensidade do periodograma para 12 meses foi menor do que para 3 e 6 meses, uma vez que o mesmo acumula variações trimestrais e semestrais. Observou-se que a série da região Norte não finaliza em ruído branco. 
Tabela 1. Coeficientes de regressão (SARIMA) de cinco taxas mensais de procedimentos odontológicos em três momentos entre julho de 1994 e julho de 2007 no Brasil.

\begin{tabular}{|c|c|c|c|c|c|c|c|}
\hline \multirow[b]{2}{*}{ Desfecho } & \multirow{2}{*}{$\begin{array}{c}\text { Intercepto } \\
\text { (por mil/hab.) } \\
\quad(\text { IC95\%) }\end{array}$} & \multicolumn{2}{|c|}{ Jan/1998 (IC95\%) } & \multicolumn{2}{|c|}{ Out/1999 (IC95\%) } & \multicolumn{2}{|c|}{ Dez/2000 (IC95\%) } \\
\hline & & $\begin{array}{c}\text { Coeficiente } \\
\text { por mil/hab. }\end{array}$ & $\begin{array}{c}\text { Percentual } \\
\text { de alteração }\end{array}$ & $\begin{array}{c}\text { Coeficiente } \\
\text { por mil/hab. }\end{array}$ & $\begin{array}{c}\text { Percentual } \\
\text { de alteração }\end{array}$ & $\begin{array}{c}\text { Coeficiente } \\
\text { por mil/hab. }\end{array}$ & $\begin{array}{c}\text { Percentual } \\
\text { de alteração }\end{array}$ \\
\hline $\begin{array}{l}\text { Taxa total de } \\
\text { procedimentos }\end{array}$ & $\begin{array}{c}65,5 \\
(57,0 / 73,9)\end{array}$ & $\begin{array}{c}-10,0 \\
(-15,4 /-4,5)\end{array}$ & $\begin{array}{c}-15,3 \% \\
(-23,5 /-6,9)\end{array}$ & $\begin{array}{c}30,5 \\
(23,6 / 37,4)\end{array}$ & $\begin{array}{c}55,0 \% \\
(42,5 / 67,4)\end{array}$ & $\begin{array}{c}5,9 \\
(0,2 / 11,7)\end{array}$ & $\begin{array}{c}6,9 \% \\
(0,3 / 13,6)\end{array}$ \\
\hline $\begin{array}{l}\text { Taxa de procedimentos } \\
\text { coletivos }\end{array}$ & $\begin{array}{c}14,5 \\
(10,4 / 18,6)\end{array}$ & $\begin{array}{c}-11,9 \\
(-16,9 /-6,9)\end{array}$ & $\begin{array}{c}-82,1 \% \\
(-116 /-47,8)\end{array}$ & $\begin{array}{c}31,6 \\
(25,6 / 37,6)\end{array}$ & $\begin{array}{c}1234 \% \\
(999 / 1469)\end{array}$ & $\begin{array}{c}5,4 \\
(0,5 / 10,4)\end{array}$ & $\begin{array}{c}15,9 \% \\
(1,6 / 30,3)\end{array}$ \\
\hline $\begin{array}{l}\text { Taxa de procedimentos } \\
\text { preventivos }\end{array}$ & $11,3^{*}$ & $\begin{array}{c}2,1 \\
(1,1 / 3,1)\end{array}$ & $\begin{array}{c}18,6 \% \\
(9,7 / 27,4)\end{array}$ & $\begin{array}{c}-0,3 \\
(-1,3 / 0,7)\end{array}$ & $\begin{array}{c}-2,4 \% \\
(-9,7 / 5,2)\end{array}$ & $\begin{array}{c}-0,9 \\
(-1,9 / 0,1)\end{array}$ & $\begin{array}{c}-6,9 \% \\
(-14,5 / 0,5)\end{array}$ \\
\hline Taxa de restaurações & $14,4^{*}$ & $\begin{array}{c}-3,0 \\
(-3,9 /-2,2)\end{array}$ & $\begin{array}{c}-20,8 \% \\
(-27,1 /-15,3)\end{array}$ & $\begin{array}{c}0,5 \\
(-0,4 / 1,4)\end{array}$ & $\begin{array}{c}4,4 \% \\
(-3,5 / 12,3)\end{array}$ & $\begin{array}{c}-0,8 \\
(-1,7 / 0,03)\end{array}$ & $\begin{array}{c}-6,7 \% \\
(-14,3 / 0,3)\end{array}$ \\
\hline Taxa de exodontias & $5,3^{*}$ & $\begin{array}{c}0,3 \\
(-0,2 / 0,9)\end{array}$ & $\begin{array}{c}5,7 \% \\
(-3,8 / 17,0)\end{array}$ & $\begin{array}{c}0,2 \\
(-0,3 / 0,7)\end{array}$ & $\begin{array}{c}3,6 \% \\
(-5,4 / 12,5)\end{array}$ & $\begin{array}{c}-0,6 \\
(-1,1 /-0,1)\end{array}$ & $\begin{array}{c}-10,3 \% \\
(-19,0 /-1,7)\end{array}$ \\
\hline
\end{tabular}

"Valores obtidos representam a estimativa suavizadas do mês imediatamente anterior a primeira intervenção, pois são séries não-estacionárias.

\section{Discussão}

O presente trabalho constitui-se de uma análise de séries temporais de procedimentos odontológicos realizados nos serviços públicos. Esses procedimentos são informados pelas Secretarias Municipais de Saúde ao Ministério da Saúde alimentando o SIA-SUS, assim a subnotificação possivelmente seja a principal limitação em termos de qualidade dos dados, não sendo possível inferir que as taxas apresentadas correspondam às verdadeiras realizadas nas unidades básicas de saúde. Ainda assim, acreditamos que isso não invalida as inferências do presente estudo para as tendências, pois um dos principais fatores que plausivelmente alteram essas séries são exatamente os fatores estudados: políticas de gestão. Outra limitação desse trabalho é a impossibilidade de se inferir tendências de procedimentos para clínicas particulares, que constituem boa parte dos prestadores de serviços.

Dentre os principais resultados, ressalta-se o decréscimo nas tendências das taxas de restaurações e exodontias, confirmando resultados de outros países. No Reino Unido ${ }^{7}$ foi relatada uma constância no número de extrações entre 1991 e 2000, entretanto, tal estudo não levou em consideração um possível crescimento no tamanho populacional. Na Austrália ${ }^{5,6}$ foi descrita uma redução no número de restaurações realizadas, bem como uma redução no número de extrações por paciente entre 1983 e 1999. No Brasil, a tendência de redução dessas taxas possivelmente reflete o declínio da cárie dental ${ }^{32-34}$, que é responsável por até $70 \%$ das extrações dentárias ${ }^{35,36}$ e pela grande maioria das restaurações. Uma possível implicação na redução das extrações é o aumento do número médio de dentes por pessoa e um aumento na taxa de restaurações, como previsto para os Estados Unidos da América ${ }^{37}$. Talvez o aumento da taxa de restaurações observado no final da série seja, em parte, reflexo da taxa decrescente de exodontias nos anos anteriores.

Um segundo importante achado desse estudo refere-se à tendência da taxa de procedimentos odontológicos totais, um indicador de uso dos serviços. O padrão dessa taxa apresentou-se estacionário, apesar da elevação entre 1998 e 2001. Em concordância com esse achado, Pinheiro e Torres ${ }^{8}$ mostraram uma redução no percentual de pessoas que nunca foram ao dentista entre os anos de 1998 e 2003. Comparações entre os dois estudos devem ser vistas com reservas, pois há importantes diferenças metodologias. Por exemplo, nosso estudo refere-se ao uso apenas dos serviços públicos. Tudo indica que, no SUS, esse aumento abrupto após 1998 não é um mero artefato de notificação, mas pode refletir um efeito tardio do processo de municipalização da saúde. O Percentual de municípios habilitados a assumir a saúde no nível local aumentou de 21,6\% em 1994 para 61,9\% em novembro 1996, superando $97 \%$ em $2000^{13}$. 
Em relação às políticas de saúde bucal, Pinheiro e Torres ${ }^{8}$ acreditam que o aumento da cobertura populacional possa ser consequência do aumento no número de novas Equipes de Saúde Bucal do PSF (ESB-PSF) e de investimentos feitos após 2001. Trabalhos recentes mostram que o impacto das ESB no PSF pode ser pequeno ${ }^{22,23}$. No presente trabalho, entre dezembro de 2000 e julho de 2007, houve um aumento médio de 6,9\% na taxa total de procedimentos em relação ao período anterior, resultante de um incremento de 5,9 procedimentos por mil habitantes (aumento expressivo apenas nas regiões Norte e Nordeste, Figura 2). O aumento após a introdução das ESBPSF é modesto, se comparado com o incremento em outubro de 1999 (possivelmente refletindo a aceleração tardia do processo de municipalização). Por outro lado, deve-se considerar que os profissionais das ESB-PSF possuem atribuições não registradas na base do SIA-SUS (i.e. visitas domiciliares, reuniões de planejamento, etc.) e pode haver também uma saturação no número de procedimentos realizados em função de limites físicos, como o número de consultórios e material disponível. Outrossim, uma mudança no uso dos serviços pode não refletir uma alteração de acesso, uma vez que as pessoas podem exercitar o direito de não usar os serviços. Acesso refere-se à oferta enquanto o uso reflete uma combinação de oferta e demanda ${ }^{11}$. Políticas que aumentam o acesso aos serviços de saúde podem não ter impacto no uso dos mesmos.

Um terceiro achado importante diz respeito às alterações no tipo de procedimentos realizados após a inclusão das ESB-PSF. A principal delas foi um aumento de $15,9 \%$ dos procedimentos coletivos. Em oposição, houve uma redução de 6,9\% dos procedimentos clínicos preventivos. Esses achados são conflitantes, uma vez que as ESBPSF não deveriam reduzir nenhuma prática preventiva existente. Pode-se especular que a saída dos cirurgiões-dentistas do ambiente clínico para visitas domiciliares e para atividades extraclínica além do necessário, seja uma explicação para esse achado. Entretanto, se isso for verdade, então reflete o inapropriado uso dos profissionais auxiliares como TSB e ASB.

Por fim, observou-se um forte padrão sazonal nas taxas totais de procedimentos odontológicos, similar em quatro das cinco regiões brasileiras. Esses padrões cíclicos parecem refletir tanto a organização dos serviços (e.g. férias anuais dos Cirurgiões-Dentistas) como a demanda (e.g. períodos escolares $)^{11}$. Os resultados apontam para uma forte repetição de padrões a cada $12 \mathrm{e}$ 6 meses.

Este estudo permite concluir que a principal alteração na série de procedimentos odontológicos coincide com o período de municipalização da saúde entre 1998 e 2000. Os impactos da inclusão de Equipes de Saúde Bucal no Programa de Saúde da Família foram variados, ressaltando-se o aumento na taxa total de procedimentos, aumento dos procedimentos coletivos e redução das exodontias. Estudos desenhados para investigar fatores que possam avaliar as atividades das ESBPSF podem ser frutíferos em auxiliar planejadores a maximizar o potencial do programa.

\section{Colaboradores}

RKC e JFV colaboraram na coleta de dados, redação e análise dos dados. WLJ participou na redação final e análise dos dados e MER participou na revisão e redação do texto. 


\section{Referências}

1. Nadanovsky P, Sheiham A. Relative contribution of dental services to the changes in caries levels of 12year-old children in 18 industrialized countries in the 1970 s and early 1980s. Community Dent Oral Epidemiol 1995; 23(6):331-339.

2. Celeste RK, Nadanovsky P, Leon AP. Association Between Public Dental Service Preventive Procedures and Caries Prevalence. Rev Saude Publica 2007; 41(5):830-838.

3. Davenport C, Elley K, Salas C, Taylor-Weetman CL, Fry-Smith A, Bryan S, Taylor R. The clinical effectiveness and cost-effectiveness of routine dental checks: a systematic review and economic evaluation. Health Technol Assess. 2003; 7(7):1-127.

4. Petersen PE, Holst D. Utilization of Dental Health Services. In: Cohen L, Gift H, editors. Disease Prevention and Oral Health Promotion: Socio-dental Sciences in Action. Copenhagen: Munksgaard; 1995. p. 341-386.

5. Brennan DS, Spencer AJ. Trends in service provision among Australian private general dental practitioners over a 20 -year period. Int Dent J 2006; 56(4):215-223.

6. Brennan DS, Spencer AJ. Restorative service trends in private general practice in Australia: 1983-1999. J Dent 2003; 31(2):143-151.

7. Dhariwal DK, Goodey R, Shepherd JR. Trends in oral surgery in England and Wales 1991-2000. Br Dent J 2002; 192(11):639-645.

8. Pinheiro R, Torres TZG. Access to oral health services between Brazilian States. Cien Saude Colet 2006; 11(4):999-1010.

9. Barros SG, Chaves SCL. Use of the Outpatient Information System (SIA-SUS) to assess oral health activities. Epidemiol serv saúde 2003; 12(1):41-51.

10. Volpato LER, Scatena JH. Analysis of the Oral Health Policy of Cuiabá Municipality, Mato Grosso State, Brazil, using the Outpatient Information System Database of the National Public Health System (SIASUS). Epidemiol serv saúde 2006; 15(2):47-55.

11. Travassos C, Martins M. A review of concepts in health services access and utilization. Cad Saude Publica 2004; 20(Supl. 2):S190-S198.

12. Andrade LOM, Pontes RJS, Martins Junior T. A descentralização no marco da Reforma Sanitária no Brasil. Rev Panam Salud Publica 2000; 8(1-2):85-91.

13. Scatena JH. Avaliação da Descentralização da Assistência a Saúde no Estado do Mato Grosso do Sul [thesis]. São Paulo: USP; 2000.

14. Silva LMV. Avaliação do processo de descentralização das ações de saúde. Cien Saude Colet 1999; $4(2): 331-339$

15. Brasil. Ministério da Saúde (MS). Portaria N.o 1.882/ GM, de 18 de dezembro de 1997. Estabelece o piso de atenção básica - PAB e sua composição. Diário Oficial da União 1997; 22 dez.

16. Silveira Filho AD. A saúde Bucal no PSF: o desafio de mudar a prática. Informe Programa Saúde na Família 2002(Dez):36-43.
17. Brasil. Ministério da Saúde (MS). Diretrizes da Politica Nacional de Saúde Bucal. 2004 [acessado 2009 mar 10]; 16 p. Disponível em: http://dtr2004.saude. gov.br/dab/docs/publicacoes/geral/diretrizes_da_ politica_nacional_de_saude_bucal.pdf

18. Brasil. Ministério da Saúde (MS). Coordenação Nacional de Saúde Bucal. Saúde da Família 2008. [acessado 2008 fev 14]. Disponível em: http:// dtr2004.saude.gov.br/dab/cnsb/saude_familia.php

19. Carvalho CL, organizador. Caracterização Dos Recursos Humanos Nos Serviços Públicos Municipais De Saúde Bucal No Brasil. Relatório Técnico. Belo Horizonte: Observatório de Recursos Humanos em Saúde/NESCOM; 2006. p. 63.

20. Baldani MH, Fadel CB, Possamai T, Queiroz MG. Inclusion of oral health services in the Family Health Program in the State of Parana, Brazil. Cad Saude Publica 2005; 21(4):1026-1035.

21. Emmi DT, Barroso RF. Evaluation of oral health actions in the Family Health Program in the Mosqueiro district, Para State, Brazil. Cien Saude Colet 2008; 13(1):35-41.

22. Pereira CR, Patricio AA, Araujo FA, Lucena EE, de Lima KC, Roncalli AG. Inclusion of oral health teams in the Family Health Program and its impact on the use of dental services. Cad Saude Publica 2009; 25(5):985-996.

23. Rocha RACP, Goes PS. Comparison of access to Oral Health Services between areas covered and not covered by the Family Health Program in Campina Grande, Paraiba State, Brazil]. Cad Saude Publica 2008; 24(12):2871-2880.

24. Laurenti R, Mello Jorge MHP, Lebrão LM, Gotlieb SLD. Estatísticas de Saúde. $2^{\text {a }}$ ed. São Paulo: Editora Pedagógica Universitária; 1987.

25. Brasil. Ministério da Saúde (MS). Portaria N. ${ }^{\circ} 1.230$ de 14 de outubro de 1999. Define os arquivos para a alimentação do Banco de Dados do Sistema de Informações Ambulatoriais do Sistema Único de Saúde - SIA/SUS. Diário Oficial da União 1999; 18 out.

26. Diggle P. Time series: a biostatistical introduction. Oxford Statistical Science Series 5. Oxford: Oxford University Press; 1990.

27. Brasil. Ministério da Saúde (MS). Instrução normativa, $n^{\circ}$ 01/98. Diário Oficial da União 1998; 02 jan.

28. Brasil. Ministério da Saúde (MS). DATASUS. Novidades na Home Page do DATASUS - $1^{\circ}$ Semestre de 1998: Discriminação das informações do SIA/SUS pelo Piso Assistencial Básico (PAB) - 16/03/1998. [acessado 2009 mar 10]. Disponível em: http:// www.datasus.gov.br/novidades/novid9801.htm

29. Brasil. Ministério da Saúde (MS). Portaria N. ${ }^{\circ} 1.444$, de 28 de dezembro de 2000. Estabelece incentivo financeiro para a reorganização da atenção à saúde bucal prestada nos municípios por meio do Programa de Saúde da Família. Diário Oficial da União. 2000; 29 dez.

30. Box GEP, Tiao GC. Intervention Analysis with Applications to Economic and Environmental Problems. Journal of the American Statistical Association. 1975; 70(349):70-79. 
31. Box GEP, Jenkins GM, Reinsel GC. Time series analysis: forecasting and control. 3rd ed. New Jersey: Prentice-Hall; 1995.

32. Brasil. Ministério da Saúde (MS). Levantamento Epidemiológico em Saúde Bucal: Brasil, zona urbana - 1986. In: Secretaria Nacional de Programas Especiais. Divisão Nacional de Saúde Bucal, organizador. Brasília: Centro de Documentação do Ministério da Saúde; 1988. p. 137.

33. Brasil. Ministério da Saúde (MS). Projeto SB Brasil 2003: condições de saúde bucal da população brasileira 2002-2003: resultados principais. In: Departamento de Atenção Básica. Secretaria de Atenção à Saúde, organizador. $1^{\text {a }}$ ed. Brasília: Coordenação Nacional de Saúde Bucal; 2004. p. 68.

34. Narvai PC, Frazao P, Roncalli AG, Antunes JL. Dental caries in Brazil: decline, polarization, inequality and social exclusion. Rev Panam Salud Publica 2006; 19(6):385-393.

35. Caldas AF, Marcenes W, Sheiham A. Reasons for tooth extraction in a Brazilian population. Int Dent $J$ 2000; 50(5):267-273.

36. Guimarães MM, Marcos B. Impact of Social Class on Tooth Extraction. Revista do CROMG 1996; II (2):78-82.

37. Douglass CW. Future needs for dental restorative materials. Adv Dent Res 1992; 6:4-6.

Artigo apresentado em 09/06/2009

Aprovado em 16/08/2009

Versão final apresentada em 25/08/2009 